TRANSACTIONS OF THE

AMERICAN MATHEMATICAL SOCIETY

Volume 355, Number 11, Pages 4639-4656

S 0002-9947(03)03326-9

Article electronically published on May 15, 2003

\title{
BAXTER ALGEBRAS AND HOPF ALGEBRAS
}

\author{
GEORGE E. ANDREWS, LI GUO, WILLIAM KEIGHER, AND KEN ONO
}

\begin{abstract}
By applying a recent construction of free Baxter algebras, we obtain a new class of Hopf algebras that generalizes the classical divided power Hopf algebra. We also study conditions under which these Hopf algebras are isomorphic.
\end{abstract}

\section{INTRODUCTION}

Hopf algebras have their origin in Hopf's seminal works on topological groups in the 1940s, and have become fundamental objects in many areas of mathematics and physics. For example, they are crucial to the study of algebraic groups, Lie groups, Lie algebras, and quantum groups. In turn, these areas have provided many of the most important examples of Hopf algebras.

In this paper we construct new examples of Hopf algebras. Our examples arise naturally in a combinatorial study of Baxter algebras. A Baxter algebra [1] is an algebra $A$ with a linear operator $P$ on $A$ that satisfies the identity

$$
P(x) P(y)=P(x P(y))+P(y P(x))+\lambda P(x y)
$$

for all $x$ and $y$ in $A$, where $\lambda$, the weight, is a fixed element in the ground ring of the algebra $A$. Rota [17 began a systematic study of Baxter algebras from an algebraic and combinatorial perspective and suggested that they are related to hypergeometric functions, incidence algebras and symmetric functions [18, 19]. A survey of Baxter algebras with examples and applications can be found in [18], [19], as well as in [7].

Free Baxter algebras were first constructed by Rota [17 and Cartier 3 in the category of Baxter algebras with no identity (with some restrictions on the weight and the base ring). Recently, two of the authors [8, 9] have constructed free Baxter algebras in a more general context including these classical constructions. Their construction is in terms of mixable shuffle products, which generalize the well-known shuffle products of path integrals as developed by Chen 4 and Ree 13 . Here we show that a special case of the construction of these new Baxter algebras provides a large supply of new Hopf algebras.

The divided power Hopf algebra is one of the classical examples of a Hopf algebra, and it is not difficult to see that this algebra is the free Baxter algebra of weight zero

Received by the editors January 24, 2003.

2000 Mathematics Subject Classification. Primary 16W30, 16W99.

Key words and phrases. Free Baxter algebra, Hopf algebra, divided power.

The first and fourth authors are supported by grants from the National Science Foundation, and the fourth author is supported by Alfred P. Sloan, David and Lucile Packard, and H. I. Romnes Fellowships. 
on the empty set. The new Hopf algebras presented here generalize this classical example. In particular, we show that the free Baxter algebra of arbitrary weight on the empty set is a Hopf algebra.

Here we describe the construction. Let $C$ be a commutative algebra with identity 1, and let $\lambda \in C$. Define the sextuple $\mathcal{A}=\mathcal{A}_{\lambda} \stackrel{\text { def }}{=}(A, \mu, \eta, \Delta, \varepsilon, S)$, where

(vi) $\quad S=S_{\lambda}: A \rightarrow A, a_{n} \mapsto(-1)^{n} \sum_{v=0}^{n}\left(\begin{array}{c}n-3 \\ v-3\end{array}\right) \lambda^{n-v} a_{v}$

Here for any positive or negative integer $x,\left(\begin{array}{l}x \\ k\end{array}\right)$ is defined by the generating function $(1+z)^{x}=\sum_{k=0}^{\infty}\left(\begin{array}{l}x \\ k\end{array}\right) z^{k}$. It was shown in 8 that $\left(A_{\lambda}, \mu_{\lambda}, \eta_{\lambda}\right)$ is a Baxter algebra of weight $\lambda$ with respect to the operator

$$
P: A_{\lambda} \rightarrow A_{\lambda}, a_{n} \mapsto a_{n+1} .
$$

The main theorem in Section 2 is

Theorem 1.1. For any $\lambda \in C, \mathcal{A}_{\lambda}$ is a Hopf $C$-algebra.

When $\lambda=0$, we have the divided power Hopf algebra. In general, $\mathcal{A}_{\lambda}$ will be called the $\lambda$-divided power Hopf algebra. The divided power algebra plays an important role in several areas of mathematics, including crystalline cohomology in number theory [2], umbral calculus in combinatorics [16] and Hurwitz series in differential algebra [10]. We expect that the $\lambda$-divided power algebra $\mathcal{A}_{\lambda}$ introduced here will play similar roles in these areas. To describe the application to umbral calculus, we recall that a sequence $\left\{p_{n}(x) \mid n \in \mathbb{N}\right\}$ of polynomials in $C[x]$ is called a sequence of binomial type if

$$
p_{n}(x+y)=\sum_{k=0}^{n}\left(\begin{array}{l}
n \\
k
\end{array}\right) p_{n-k}(x) p_{k}(y)
$$

in $C[x, y]$. The classic umbral calculus studies these sequences and their generalizations, such as Sheffer sequences and cross sequences. It is well known that the theory of classic umbral calculus can be most conceptually described in the framework of Hopf algebras. Furthermore, most of the main results in umbral calculus follow from properties of the divided power Hopf algebra structure on the linear 
dual $\operatorname{Hom}_{C}(C[x], C)$, usually called the umbral algebra (see [12, [15] for details). This algebra is the completion of the divided power algebra equipped with an umbral shift operator which turns out to be a Baxter operator, making the umbral algebra the complete free Baxter algebra of weight zero on the empty set. See [6] for details. A program carried out there gives a generalization of the umbral calculus using the $\lambda$-divided power algebra.

It is natural to ask whether these Hopf algebras are isomorphic for different values of $\lambda$. We address this question in Section 3 and obtain the following result.

Theorem 1.2. Let $\lambda, \nu$ be in $C$.

(1) If $(\lambda)=(\nu)$, then $\mathcal{A}_{\lambda}$ and $\mathcal{A}_{\nu}$ are isomorphic Hopf algebras.

(2) Suppose $C$ is a $\mathbb{Q}$-algebra. If $\mathcal{A}_{\lambda}$ and $\mathcal{A}_{\nu}$ are isomorphic Hopf algebras, then $(\lambda)=(\nu)$.

(3) If $\mathcal{A}_{\lambda}$ and $\mathcal{A}_{\nu}$ are isomorphic Hopf algebras, then $\sqrt{(\lambda)}=\sqrt{(\nu)}$.

We also prove a stronger but more technical version of the third statement in Proposition 3.4

According to Theorem 1.2 for any $C$, there are at least two non-isomorphic $\lambda$ divided power Hopf algebras, namely $\mathcal{A}_{0}$ and $\mathcal{A}_{1}$. Furthermore, if $C$ is a $\mathbb{Q}$-algebra, then there is a one-to-one correspondence between isomorphic classes of $\lambda$-divided power Hopf algebras and principal ideals of $C$. For other examples, see Example 3.5

\section{ON $\lambda$-DIVIDED POWER HOPF ALGEBRAS}

Since the constant $\lambda \in C$ will be fixed throughout this section, we will drop the dependence on the subscript $\lambda$. In section 2.1 we recall the defining properties of a Hopf algebra. The remainder of the section establishes that $\mathcal{A}_{\lambda}$ satisfies these properties. Since two of the authors proved that $(A, \mu, \eta)$ is a $C$-algebra $[8$, the proof of Theorem 1.1 reduces to a step-by-step verification of the defining properties of a Hopf algebra.

2.1. Preliminaries. Here we recall some basic definitions and facts for later reference. All tensor products in this paper are taken over the fixed commutative ring $C$. Recall that a cocommutative $C$-coalgebra is a triple $(A, \Delta, \varepsilon)$ where $A$ is a $C$-module, $\Delta: A \rightarrow A \otimes A$ and $\varepsilon: A \rightarrow C$ are $C$-linear maps that make the following diagrams commute:

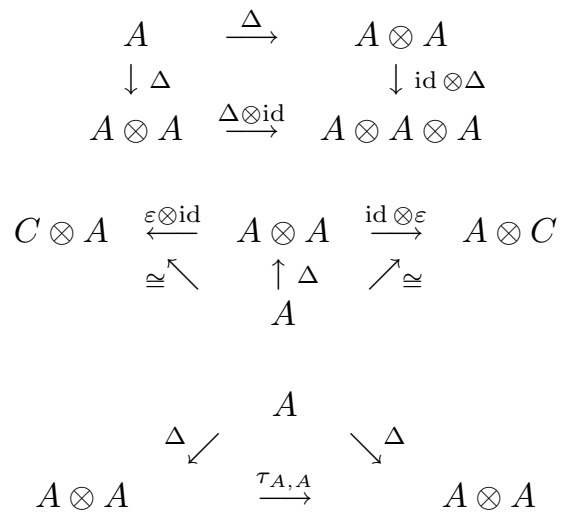


where $\tau_{A, A}: A \otimes A \rightarrow A \otimes A$ is defined by $\tau_{A, A}(x \otimes y)=y \otimes x$. The $C$-algebra $C$ has a natural structure of a $C$-coalgebra with

$$
\Delta_{C}: C \rightarrow C \otimes C, c \mapsto c \otimes 1, c \in C
$$

and

$$
\varepsilon_{C}=\mathrm{id}_{C}: C \rightarrow C .
$$

We also denote the multiplication in $C$ by $\mu_{C}$.

Recall that a $C$-bialgebra is a quintuple $(A, \mu, \eta, \Delta, \varepsilon)$ where $(A, \mu, \eta)$ is a $C$ algebra and $(A, \Delta, \varepsilon)$ is a $C$-coalgebra such that $\mu$ and $\eta$ are morphisms of $C$ coalgebras. In other words, we have the commutativity of the following diagrams.
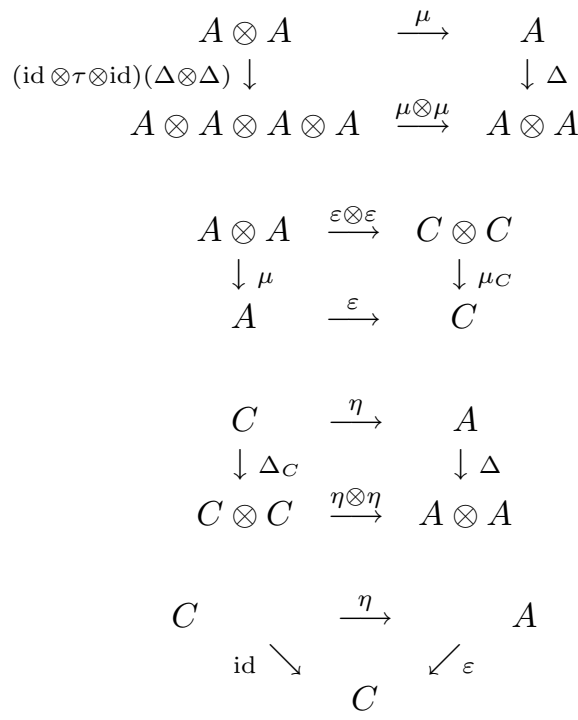

Let $(A, \mu, \eta, \Delta, \varepsilon)$ be a $C$-bialgebra. For $C$-linear maps $f, g: A \rightarrow A$, the convolution $f \star g$ of $f$ and $g$ is the composition of the maps

$$
A \stackrel{\Delta}{\longrightarrow} A \otimes A \stackrel{f \otimes g}{\longrightarrow} A \otimes A \stackrel{\mu}{\longrightarrow} A .
$$

A $C$-linear endomorphism $S$ of $A$ is called an antipode for $A$ if

$$
S \star \operatorname{id}_{A}=\operatorname{id}_{A} \star S=\eta \circ \varepsilon .
$$

A Hopf algebra is a bialgebra $A$ with an antipode $S$.

2.2. Coalgebra Properties. We verify that $(A, \Delta, \varepsilon)$ satisfies the axioms of a coalgebra characterized by the diagrams (2.1), (2.2) and (2.3).

To prove (2.1), we only need to verify that, for each $n \geq 0$,

$$
(\Delta \otimes \mathrm{id})\left(\Delta\left(a_{n}\right)\right)=(\mathrm{id} \otimes \Delta)\left(\Delta\left(a_{n}\right)\right) .
$$

Unwinding definitions on the left-hand side we get

$$
(\Delta \otimes \mathrm{id})\left(\Delta\left(a_{n}\right)\right)=\sum_{k=0}^{n} \sum_{i=0}^{n-k} \sum_{\ell=0}^{i} \sum_{j=0}^{i-\ell}(-\lambda)^{k+\ell} a_{j} \otimes a_{i-\ell-j} \otimes a_{n-k-i} .
$$


Exchanging the third and the fourth summations, and then exchanging the second and the third summations, we obtain

$$
(\Delta \otimes \mathrm{id})\left(\Delta\left(a_{n}\right)\right)=\sum_{k=0}^{n} \sum_{j=0}^{n-k} \sum_{i=j}^{n-k} \sum_{\ell=0}^{i-j}(-\lambda)^{k+\ell} a_{j} \otimes a_{i-\ell-j} \otimes a_{n-k-i} .
$$

Replacing $i$ by $i+j$ gives us

$$
(\Delta \otimes \mathrm{id})\left(\Delta\left(a_{n}\right)\right)=\sum_{k=0}^{n} \sum_{j=0}^{n-k} \sum_{i=0}^{n-k-j} \sum_{\ell=0}^{i}(-\lambda)^{k+\ell} a_{j} \otimes a_{i-\ell} \otimes a_{n-k-i-j} .
$$

Exchanging the third and the fourth summations, followed by a substitution of $i$ by $i+\ell$, gives us

$$
(\Delta \otimes \mathrm{id})\left(\Delta\left(a_{n}\right)\right)=\sum_{k=0}^{n} \sum_{j=0}^{n-k} \sum_{\ell=0}^{n-k-j} \sum_{i=0}^{n-k-j-\ell}(-\lambda)^{k+\ell} a_{j} \otimes a_{i} \otimes a_{n-k-i-\ell-j} .
$$

We get the same expression after unwinding definitions on the right-hand side. This proves equation (2.9).

Before proving the commutativity of diagram (2.2) we display a lemma.

Lemma 2.1. For any integers $n$ and $\ell$ with $n \geq \ell \geq 0$, we have

$$
\sum_{k=0}^{n-\ell}(-\lambda)^{k} \varepsilon\left(a_{n-\ell-k}\right)= \begin{cases}\mathbf{1}, & \ell=n, \\ 0, & \ell<n .\end{cases}
$$

Proof. When $\ell=n$, we have

$$
\sum_{k=0}^{n-\ell}(-\lambda)^{k} \varepsilon\left(a_{n-\ell-k}\right)=\varepsilon\left(a_{0}\right)=\mathbf{1} .
$$

When $\ell<n$, we have

$$
\sum_{k=0}^{n-\ell}(-\lambda)^{k} \varepsilon\left(a_{n-\ell-k}\right)=(-\lambda)^{n-\ell-1} \varepsilon\left(a_{1}\right)+(-\lambda)^{n-\ell} \varepsilon\left(a_{0}\right) .
$$

By the definition of $\varepsilon\left(a_{n}\right)$, the right-hand side is

$$
(-\lambda)^{n-\ell-1} \lambda \mathbf{1}+(-\lambda)^{n-\ell} \mathbf{1}=0 .
$$

We can now prove (2.2). Consider the left triangle in (2.2). For each $n \geq 0$, we have

$$
(\varepsilon \otimes \mathrm{id})\left(\Delta\left(a_{n}\right)\right)=\sum_{k=0}^{n} \sum_{i=0}^{n-k}(-\lambda)^{k} \varepsilon\left(a_{i}\right) \otimes a_{n-k-i} .
$$

A substitution $i=n-k-\ell$ and then an exchange of the order of summations give us

$$
(\varepsilon \otimes \mathrm{id})\left(\Delta\left(a_{n}\right)\right)=\sum_{\ell=0}^{n}\left(\sum_{k=0}^{n-\ell}(-\lambda)^{k} \varepsilon\left(a_{n-k-\ell}\right)\right) \otimes a_{\ell},
$$


which is $1 \otimes a_{n}$ by Lemma 2.1. This proves the commutativity of the left triangle in (2.2).

The proof of the right triangle in (2.2) is similar.

The cocommutativity of diagram (2.3) is easy to verify:

$$
\tau_{A, A}\left(\Delta\left(a_{n}\right)\right)=\sum_{k=0}^{n} \sum_{i=0}^{n-k}(-\lambda)^{k} a_{n-k-i} \otimes a_{i} .
$$

After replacing $i$ by $n-k-j$, we see that it is the same as $\Delta\left(a_{n}\right)$.

Hence we have shown that $(A, \Delta, \varepsilon)$ is a cocommutative $C$-coalgebra.

2.3. Compatibility. We now prove that the algebra and coalgebra structures on $A$ are compatible so that they give a bialgebra structure on $A$.

Since

$$
\varepsilon(\eta(\mathbf{1}))=\varepsilon(\mathbf{1})=\varepsilon\left(a_{0}\right)=\mathbf{1}=\mathrm{id}(\mathbf{1}),
$$

we have verified the commutativity of diagram (2.7).

We also have

$$
(\eta \otimes \eta)\left(\Delta_{C}(\mathbf{1})\right)=(\eta \otimes \eta)(\mathbf{1} \otimes \mathbf{1})=a_{0} \otimes a_{0}
$$

and

$$
\Delta(\eta(\mathbf{1}))=\Delta\left(a_{0}\right)=\sum_{k=0}^{0} \sum_{i=0}^{0-k}(-\lambda)^{k} a_{i} \otimes a_{0-k-i}=a_{0} \otimes a_{0} .
$$

This proves the commutativity of diagram (2.6).

We next prove the commutativity of diagram (2.5). We have

$$
(\varepsilon \otimes \varepsilon)\left(a_{m} \otimes a_{n}\right)= \begin{cases}\mathbf{1} \otimes \mathbf{1}, & (m, n)=(0,0), \\ \lambda \mathbf{1} \otimes \mathbf{1}, & (m, n)=(1,0), \\ \mathbf{1} \otimes \lambda \mathbf{1}, & (m, n)=(0,1), \\ \lambda \mathbf{1} \otimes \lambda \mathbf{1}, & (m, n)=(1,1), \\ 0, & m \geq 2 \text { or } n \geq 2 .\end{cases}
$$

So

$$
\mu_{C}\left((\varepsilon \otimes \varepsilon)\left(a_{m} \otimes a_{n}\right)\right)= \begin{cases}\mathbf{1}, & (m, n)=(0,0), \\ \lambda \mathbf{1}, & (m, n)=(1,0) \text { or }(0,1), \\ \lambda^{2} \mathbf{1}, & (m, n)=(1,1), \\ 0, & m \geq 2 \text { or } n \geq 2 .\end{cases}
$$

On the other hand, we have

$$
\begin{aligned}
\varepsilon\left(\mu\left(a_{m} \otimes a_{n}\right)\right) & =\varepsilon\left(\sum_{i=0}^{m} \lambda^{i}\left(\begin{array}{c}
m+n-i \\
m
\end{array}\right)\left(\begin{array}{c}
m \\
i
\end{array}\right) a_{m+n-i}\right) \\
& =\sum_{i=0}^{m} \lambda^{i}\left(\begin{array}{c}
m+n-i \\
m
\end{array}\right)\left(\begin{array}{c}
m \\
i
\end{array}\right) \varepsilon\left(a_{m+n-i}\right) .
\end{aligned}
$$

So when $(m, n)=(0,0)$, we have

$$
\varepsilon\left(\mu\left(a_{0} \otimes a_{0}\right)\right)=\varepsilon\left(a_{0}\right)=\mathbf{1} .
$$

When $(m, n)=(0,1)$, we have

$$
\varepsilon\left(\mu\left(a_{0} \otimes a_{1}\right)\right)=\sum_{i=0}^{0} \lambda^{i}\left(\begin{array}{c}
1-i \\
0
\end{array}\right)\left(\begin{array}{l}
0 \\
i
\end{array}\right) \varepsilon\left(a_{1-i}\right)=\varepsilon\left(a_{1}\right)=\lambda \mathbf{1} .
$$


By the commutativity of the multiplication $\mu$ in $A$, we also have

$$
\varepsilon\left(\mu\left(a_{1} \otimes a_{0}\right)\right)=\lambda \mathbf{1} .
$$

When $(m, n)=(1,1)$, we have

$$
\begin{aligned}
\varepsilon\left(\mu\left(a_{1} \otimes a_{1}\right)\right) & =\sum_{i=0}^{1} \lambda^{i}\left(\begin{array}{c}
2-i \\
1
\end{array}\right)\left(\begin{array}{l}
1 \\
i
\end{array}\right) \varepsilon\left(a_{2-i}\right) \\
& =\lambda^{0}\left(\begin{array}{l}
2 \\
1
\end{array}\right)\left(\begin{array}{l}
1 \\
0
\end{array}\right) \varepsilon\left(a_{2}\right)+\lambda\left(\begin{array}{l}
1 \\
1
\end{array}\right)\left(\begin{array}{l}
1 \\
1
\end{array}\right) \varepsilon\left(a_{1}\right) \\
& =\lambda^{2} \mathbf{1}
\end{aligned}
$$

When $n \geq 2$, we have $m+n-i \geq 2$ for $0 \leq i \leq m$. Thus $\varepsilon\left(a_{m+n-i}\right)=0$ and $\varepsilon\left(\mu\left(a_{m} \otimes a_{n}\right)\right)=0$. The same is true when $m \geq 2$ by the commutativity of $\mu$.

Thus we have verified the commutativity of diagram (2.5).

The rest of this section is devoted to the verification of the commutativity of diagram (2.4). In other words, we want to prove the identity

$$
\Delta\left(\mu\left(a_{m} \otimes a_{n}\right)\right)=(\mu \otimes \mu)\left(\mathrm{id} \otimes \tau_{A, A} \otimes \mathrm{id}\right)(\Delta \otimes \Delta)\left(a_{m} \otimes a_{n}\right),
$$

for any $m, n \geq 0$. The left-hand side can be simplified as follows:

$$
\begin{aligned}
\sum_{i=0}^{m} & \sum_{k=0}^{m+n-i} \sum_{j=0}^{m+n-i-k}(-1)^{k} \lambda^{i+k}\left(\begin{array}{c}
m+n-i \\
m
\end{array}\right)\left(\begin{array}{c}
m \\
i
\end{array}\right) a_{j} \otimes a_{m+n-i-k-j} \\
= & \sum_{i=0}^{m+n} \sum_{k=0}^{m+n-i} \sum_{j=0}^{m+n-i-k}(-1)^{k} \lambda^{i+k}\left(\begin{array}{c}
m+n-i \\
m
\end{array}\right)\left(\begin{array}{c}
m \\
i
\end{array}\right) a_{j} \otimes a_{m+n-i-k-j} \\
& \left(\left(\begin{array}{c}
m \\
i
\end{array}\right)=0 \text { for } i>m\right) \\
= & \sum_{j=0}^{m+n} \sum_{k=0}^{m+n-j} \sum_{i=0}^{m+n-j-k}(-1)^{k} \lambda^{i+k}\left(\begin{array}{c}
m+n-i \\
m
\end{array}\right)\left(\begin{array}{c}
m \\
i
\end{array}\right) a_{j} \otimes a_{m+n-i-k-j}
\end{aligned}
$$

(exchanging the first and third summations)

$$
\begin{aligned}
= & \sum_{j=0}^{m+n} \sum_{k=0}^{m+n-i} \sum_{\ell=0}^{m+n-j-k}(-1)^{k} \lambda^{m+n-j-\ell}\left(\begin{array}{c}
j+k+\ell \\
m
\end{array}\right)\left(\begin{array}{c}
m \\
m+n-j-k-\ell
\end{array}\right) a_{j} \otimes a_{\ell} \\
& (\text { letting } i=m+n-j-k-\ell \text { in the third sum }) \\
= & \sum_{j=0}^{m+n} \sum_{\ell=0}^{m+n-j} \lambda^{m+n-j-\ell}\left(\sum_{k=0}^{m+n-j-\ell}(-1)^{k}\left(\begin{array}{c}
j+k+\ell \\
m
\end{array}\right)\left(\begin{array}{c}
m \\
m+n-j-k-\ell
\end{array}\right)\right) a_{j} \otimes a_{\ell}
\end{aligned}
$$

(exchanging the second and third summations).

We next simplify the right-hand side of equation 2.11). Unwinding definitions we see that the right-hand side is

$$
\begin{aligned}
\sum_{k=0}^{m} & \sum_{i=0}^{m-k} \sum_{\ell=0}^{n} \sum_{j=0}^{n-\ell} \sum_{u=0}^{i} \sum_{v=0}^{m-k-i}(-1)^{k+\ell} \lambda^{k+\ell+u+v} \\
& \times\left(\begin{array}{c}
i+j-u \\
i
\end{array}\right)\left(\begin{array}{c}
i \\
u
\end{array}\right)\left(\begin{array}{c}
m+n-k-i-\ell-j-v \\
m-k-i
\end{array}\right)\left(\begin{array}{c}
m-k-i \\
v
\end{array}\right) a_{i+j-u} \otimes a_{m+n-k-i-\ell-j-v} .
\end{aligned}
$$


By substitutions

$$
\left\{\begin{array}{l}
b=i+j-u \\
e=m+n-k-i-j-\ell-v
\end{array}\right.
$$

where we treat $u$ and $v$ as the variables, we obtain

$$
\begin{aligned}
\sum_{k=0}^{m} & \sum_{i=0}^{m-k} \sum_{\ell=0}^{n} \sum_{j=0}^{n-\ell} \sum_{b=j}^{i+j} \sum_{e=n-\ell-j}^{n+m-i-j-k-\ell}(-1)^{k+\ell} \lambda^{m+n-b-e} \\
& \times\left(\begin{array}{c}
b \\
i
\end{array}\right)\left(\begin{array}{c}
i \\
i+j-b
\end{array}\right)\left(\begin{array}{c}
e \\
m-k-i
\end{array}\right)\left(\begin{array}{c}
m-k-i \\
m+n-i-j-k-\ell-e
\end{array}\right) a_{b} \otimes a_{e} .
\end{aligned}
$$

Because of the nature of the summation limits, we cannot yet exchange the order of the summations as we did for the left-hand side of equation (2.11). But we have

Lemma 2.2.

$$
\begin{aligned}
& \sum_{k=0}^{m} \sum_{i=0}^{m-k} \sum_{\ell=0}^{n} \sum_{j=0}^{n-\ell} \sum_{b=j}^{i+j} \sum_{e=n-\ell-j}^{n+m-i-j-k-\ell}(-1)^{k+\ell} \lambda^{m+n-b-e} \\
& \quad \times\left(\begin{array}{c}
b \\
i
\end{array}\right)\left(\begin{array}{c}
i \\
i+j-b
\end{array}\right)\left(\begin{array}{c}
e \\
m-k-i
\end{array}\right)\left(\begin{array}{c}
m-k-i \\
m+n-i-j-k-\ell-e
\end{array}\right) a_{b} \otimes a_{e} \\
& =\sum_{k=0}^{m+n} \sum_{i=0}^{m+n} \sum_{\ell=0}^{m+n} \sum_{j=0}^{m+n} \sum_{b=0}^{m+n} \sum_{e=0}^{m+n}(-1)^{k+\ell} \lambda^{m+n-b-e} \\
& \quad \times\left(\begin{array}{c}
b \\
i
\end{array}\right)\left(\begin{array}{c}
i \\
i+j-b
\end{array}\right)\left(\begin{array}{c}
e \\
m-k-i
\end{array}\right)\left(\begin{array}{c}
m-k-i \\
m+n-i-j-k-\ell-e
\end{array}\right) a_{b} \otimes a_{e} .
\end{aligned}
$$

Proof. Note that we have $m, n, b, e \geq 0$ by assumption. Also for any integers $x, y$ with $x \geq 0$ and $y<0$ or $x \geq 0$ and $y>x$, we have $\left(\begin{array}{l}x \\ y\end{array}\right)=0$. So

$$
k>m \Rightarrow m-k-i<0 \Rightarrow\left(\begin{array}{c}
e \\
m-k-i
\end{array}\right)=0 .
$$

This shows that we can replace the first sum on the left-hand side of the equation in the lemma by the first sum on the right-hand side. Similarly, we have

$$
\begin{aligned}
& i>m-k \quad \Rightarrow m-k-i<0 \Rightarrow\left(\begin{array}{c}
e \\
m-k-i
\end{array}\right)=0, \\
& \ell>n \quad \Rightarrow n-\ell-j<0 \Rightarrow\left(\begin{array}{c}
e \\
n-\ell-j
\end{array}\right)=0, \\
& j>n-\ell \quad \Rightarrow n-\ell-j<0 \Rightarrow\left(\begin{array}{c}
e \\
n-\ell-j
\end{array}\right)=0 \text {, } \\
& b<j \quad \Rightarrow \quad\left(\begin{array}{l}
b \\
j
\end{array}\right)=0, \\
& b>i+j \quad \Rightarrow i+j-b<0 \Rightarrow\left(\begin{array}{c}
i \\
i+j-b
\end{array}\right)=0, \\
& e<n-j-\ell \quad \Rightarrow \quad\left(\begin{array}{c}
e \\
n-j-\ell
\end{array}\right)=0, \\
& e>m+n-i-j-k-\ell \Rightarrow\left(\begin{array}{c}
m-k-i \\
m+n-i-j-k-\ell-e
\end{array}\right)=0 .
\end{aligned}
$$

Considering, in addition,

$$
\left(\begin{array}{l}
b \\
i
\end{array}\right)\left(\begin{array}{c}
i \\
i+j-b
\end{array}\right)=\left(\begin{array}{l}
b \\
j
\end{array}\right)\left(\begin{array}{c}
j \\
i+j-b
\end{array}\right)
$$


and

$$
\left(\begin{array}{c}
e \\
m-k-i
\end{array}\right)\left(\begin{array}{c}
m-k-i \\
m+n-i-j-k-\ell-e
\end{array}\right)=\left(\begin{array}{c}
e \\
n-\ell-j
\end{array}\right)\left(\begin{array}{c}
n-\ell-j \\
m+n-i-j-k-\ell-e
\end{array}\right),
$$

we see that each of the other sums on the left-hand side of the equation can be replaced by the corresponding sum on the right-hand side of the equation. This proves the lemma.

Continuing with the proof of the commutativity of the diagram (2.4), we see that the limits of the sums on the right-hand side of the equation in Lemma 2.2 are given by the same constants. Thus we can exchange the order of the summations and get

$$
\begin{aligned}
& \sum_{k=0}^{m+n} \sum_{i=0}^{m+n} \sum_{\ell=0}^{m+n} \sum_{j=0}^{m+n} \sum_{b=0}^{m+n} \sum_{e=0}^{m+n}(-1)^{k+\ell} \lambda^{m+n-b-e} \\
& \quad \times\left(\begin{array}{c}
b \\
i
\end{array}\right)\left(\begin{array}{c}
i \\
i+j-b
\end{array}\right)\left(\begin{array}{c}
e \\
m-k-i
\end{array}\right)\left(\begin{array}{c}
m-k-i \\
m+n-i-j-k-\ell-e
\end{array}\right) a_{b} \otimes a_{e} \\
& =\sum_{b=0}^{m+n} \sum_{e=0}^{m+n} \sum_{i=0}^{m+n} \sum_{j=0}^{m+n} \sum_{k=0}^{m+n} \sum_{\ell=0}^{m+n}(-1)^{k+\ell} \lambda^{m+n-b-e} \\
& \quad \times\left(\begin{array}{c}
b \\
i
\end{array}\right)\left(\begin{array}{c}
i \\
i+j-b
\end{array}\right)\left(\begin{array}{c}
e \\
m-k-i
\end{array}\right)\left(\begin{array}{c}
m-k-i \\
m+n-i-j-k-\ell-e
\end{array}\right) a_{b} \otimes a_{e} .
\end{aligned}
$$

Now by the same argument as in the proof of Lemma 2.2 we obtain

$$
\begin{aligned}
& \sum_{b=0}^{m+n} \sum_{e=0}^{m+n} \sum_{k=0}^{m+n} \sum_{i=0}^{m+n} \sum_{\ell=0}^{m+n} \sum_{j=0}^{m+n}(-1)^{k+\ell} \lambda^{m+n-b-e} \\
& \quad \times\left(\begin{array}{c}
b \\
i
\end{array}\right)\left(\begin{array}{c}
i \\
i+j-b
\end{array}\right)\left(\begin{array}{c}
e \\
m-k-i
\end{array}\right)\left(\begin{array}{c}
m-k-i \\
m+n-i-j-k-\ell-e
\end{array}\right) a_{b} \otimes a_{e} \\
& =\sum_{b=0}^{m+n} \sum_{e=0}^{m+n-b}\left[\sum_{i=0}^{b} \sum_{j=b-i}^{b} \sum_{k=0}^{m-i} \sum_{\ell=0}^{m+n-i-j-k-e}(-1)^{k+\ell} \lambda^{m+n-b-e}\right. \\
& \left.\quad \times\left(\begin{array}{c}
b \\
i
\end{array}\right)\left(\begin{array}{c}
i \\
i+j-b
\end{array}\right)\left(\begin{array}{c}
e \\
m-k-i
\end{array}\right)\left(\begin{array}{c}
m-k-i \\
m+n-i-j-k-\ell-e
\end{array}\right)\right] a_{b} \otimes a_{e} .
\end{aligned}
$$

Comparing the right-hand side of the above equation with the simplified form of the left-hand side of equation (2.11), we see that to prove equation (2.11), we only need to prove

$$
\begin{aligned}
& \sum_{k=0}^{m+n-b-e}(-1)^{k}\left(\begin{array}{c}
b+e+k \\
m
\end{array}\right)\left(\begin{array}{c}
m \\
m+n-b-e-k
\end{array}\right) \\
= & \sum_{i=0}^{b} \sum_{j=b-i}^{b} \sum_{k=0}^{m-i} \sum_{\ell=0}^{m+n-i-j-k-e}(-1)^{k+\ell}\left(\begin{array}{l}
b \\
i
\end{array}\right)\left(\begin{array}{c}
i \\
i+j-b
\end{array}\right)\left(\begin{array}{c}
e \\
m-k-i
\end{array}\right)\left(\begin{array}{c}
m-k-i \\
m+n-i-j-k-\ell-e
\end{array}\right)
\end{aligned}
$$

for all $m, n, b, e \geq 0$ with $b+e \leq m+n$. 
Using the substitutions

$$
\left\{\begin{array}{l}
j=b-i+c, \\
k=m-i-a, \\
\ell=n-b-c-e+i+a-d
\end{array}\right.
$$

on the right-hand side of equation (2.12) gives us

$$
\begin{aligned}
& \sum_{i=0}^{b} \sum_{j=b-i}^{b} \sum_{k=0}^{m-i} \sum_{\ell=0}^{m+n-i-j-k-e}(-1)^{k+\ell}\left(\begin{array}{l}
b \\
i
\end{array}\right)\left(\begin{array}{c}
i \\
i+j-b
\end{array}\right)\left(\begin{array}{c}
e \\
m-k-i
\end{array}\right)\left(\begin{array}{c}
m-k-i \\
m+n-i-j-k-\ell-e
\end{array}\right) \\
& =\sum_{i=0}^{b} \sum_{c=0}^{i} \sum_{a=0}^{m-i n-b-c-e+i+a} \sum_{d=0}^{m}(-1)^{m+n-e-b-c-d}\left(\begin{array}{l}
b \\
i
\end{array}\right)\left(\begin{array}{l}
i \\
c
\end{array}\right)\left(\begin{array}{l}
e \\
a
\end{array}\right)\left(\begin{array}{l}
a \\
d
\end{array}\right) .
\end{aligned}
$$

Thus to prove equation (2.12), and hence equation (2.11), we only need to prove the following theorem.

Theorem 2.3. If $m, n, b$ and $e$ are nonnegative integers satisfying

$$
m+n \geq b+e,
$$

then

$$
\begin{array}{r}
(-1)^{m+n-e-b} \sum_{k=0}^{m+n-b-e}(-1)^{k}\left(\begin{array}{c}
b+e+k \\
m
\end{array}\right)\left(\begin{array}{c}
m \\
m+n-b-e-k
\end{array}\right) \\
=\sum_{i=0}^{b} \sum_{c=0}^{i} \sum_{a=0}^{m-i} \sum_{d=0}^{n-e-b+i-c+a}(-1)^{c+d}\left(\begin{array}{l}
b \\
i
\end{array}\right)\left(\begin{array}{l}
i \\
c
\end{array}\right)\left(\begin{array}{l}
e \\
a
\end{array}\right)\left(\begin{array}{l}
a \\
d
\end{array}\right) .
\end{array}
$$

Proof. By replacing $k$ by $m+n-b-e-k$ in the sum on the left-hand side of the above equation and using the fact that [20]

$$
\sum_{d=0}^{j}(-1)^{d}\left(\begin{array}{l}
a \\
d
\end{array}\right)=(-1)^{j}\left(\begin{array}{c}
a-1 \\
j
\end{array}\right)
$$

(notice that this means that $\left.\left(\begin{array}{c}-1 \\ j\end{array}\right)=(-1)^{j}\right)$, the problem is reduced to showing that

$$
\begin{gathered}
\sum_{i=0}^{b} \sum_{c=0}^{i} \sum_{a=0}^{m-i}(-1)^{n-e-b+i+a}\left(\begin{array}{l}
b \\
i
\end{array}\right)\left(\begin{array}{l}
i \\
c
\end{array}\right)\left(\begin{array}{l}
e \\
a
\end{array}\right)\left(\begin{array}{c}
a-1 \\
n-e-b+i-c+a
\end{array}\right) \\
=\sum_{k=0}^{m+n-b-e}(-1)^{k}\left(\begin{array}{c}
m+n-k \\
m
\end{array}\right)\left(\begin{array}{c}
m \\
k
\end{array}\right) .
\end{gathered}
$$

Using the classical summation of Vandermonde [20,

$$
\sum_{k=0}^{m}\left(\begin{array}{c}
m \\
k
\end{array}\right)\left(\begin{array}{c}
n \\
i-k
\end{array}\right)=\left(\begin{array}{c}
m+n \\
i
\end{array}\right)
$$

in the summation on $c$ on the left-hand side of (2.13), we find that (2.13) reduces to 


$$
\begin{gathered}
\sum_{i=0}^{b} \sum_{a=0}^{m-i}(-1)^{n-e-b+i+a}\left(\begin{array}{c}
b \\
i
\end{array}\right)\left(\begin{array}{c}
e \\
a
\end{array}\right)\left(\begin{array}{c}
a+i-1 \\
n-e-b+i+a
\end{array}\right) \\
=\sum_{k=0}^{m+n-b-e}(-1)^{k}\left(\begin{array}{c}
m+n-k \\
m
\end{array}\right)\left(\begin{array}{c}
m \\
k
\end{array}\right) .
\end{gathered}
$$

Now set $T=a+i$. By the Vandermonde summation again, we find that the left-hand side of (2.14) becomes

$$
\begin{gathered}
\sum_{T \leq m} \sum_{i=0}^{b}(-1)^{n-e-b+T}\left(\begin{array}{c}
b \\
i
\end{array}\right)\left(\begin{array}{c}
e \\
T-i
\end{array}\right)\left(\begin{array}{c}
T-1 \\
n-e-b+T
\end{array}\right) \\
=\sum_{T \leq m}(-1)^{n-e-b+T}\left(\begin{array}{c}
b+e \\
T
\end{array}\right)\left(\begin{array}{c}
T-1 \\
n-e-b+T
\end{array}\right) .
\end{gathered}
$$

Therefore, it suffices to prove, by letting $H=b+e$ in (2.14), the following identity:

$$
\begin{aligned}
& \sum_{k=0}^{m+n-H}(-1)^{k}\left(\begin{array}{c}
m+n-k \\
m
\end{array}\right)\left(\begin{array}{c}
m \\
k
\end{array}\right) \\
& \quad=\sum_{T \leq m}(-1)^{n-H+T}\left(\begin{array}{c}
H \\
T
\end{array}\right)\left(\begin{array}{c}
T-1 \\
n-H+T
\end{array}\right) .
\end{aligned}
$$

For brevity, write (2.15) as

$$
L(m, n)=R(m, n) .
$$

Clearly we have the following:

$$
L(m, 0)=L(0, n)=R(0, n)=R(m, 0)=1,
$$

$$
R(m, n)-R(m-1, n)=(-1)^{n+m-H}\left(\begin{array}{c}
H \\
m
\end{array}\right)\left(\begin{array}{c}
m-1 \\
n+m-H
\end{array}\right) \text {. }
$$

Therefore, we have

$$
\begin{aligned}
& R(m, n)-R(m-1, n)-R(m, n-1)+R(m-1, n-1) \\
& \quad=(-1)^{n+m-H}\left(\begin{array}{c}
H \\
m
\end{array}\right)\left(\left(\begin{array}{c}
m-1 \\
n+m-H
\end{array}\right)+\left(\begin{array}{c}
m-1 \\
n-1+m-H
\end{array}\right)\right) \\
& \quad=(-1)^{n+m-H}\left(\begin{array}{c}
H \\
m
\end{array}\right)\left(\begin{array}{c}
m \\
n+m-H
\end{array}\right) .
\end{aligned}
$$

Using the fact that

$$
\left(\begin{array}{c}
m+n-k \\
m
\end{array}\right)\left(\begin{array}{c}
m \\
k
\end{array}\right)=\left(\begin{array}{c}
n \\
k
\end{array}\right)\left(\begin{array}{c}
m+n-k \\
n
\end{array}\right),
$$


we find that

$$
\begin{aligned}
& L(m, n)-L(m-1, n) \\
& =(-1)^{m+n-H}\left(\begin{array}{c}
H \\
m
\end{array}\right)\left(\begin{array}{c}
m \\
m+n-H
\end{array}\right) \\
& +\sum_{k=0}^{m+n-H-1}(-1)^{k}\left(\begin{array}{c}
n \\
k
\end{array}\right)\left(\left(\begin{array}{c}
m+n-k \\
n
\end{array}\right)-\left(\begin{array}{c}
m+n-k-1 \\
n
\end{array}\right)\right) \\
& =(-1)^{m+n-H}\left(\begin{array}{c}
H \\
m
\end{array}\right)\left(\begin{array}{c}
m \\
m+n-H
\end{array}\right) \\
& +\sum_{k=0}^{m+n-H-1}(-1)^{k}\left(\begin{array}{c}
n \\
k
\end{array}\right)\left(\begin{array}{c}
m+n-k-1 \\
m-k
\end{array}\right) \\
& =(-1)^{m+n-H}\left(\begin{array}{c}
H \\
m
\end{array}\right)\left(\begin{array}{c}
m \\
m+n-H
\end{array}\right) \\
& +\sum_{k=0}^{m+n-H-1}(-1)^{k}\left(\left(\begin{array}{c}
n-1 \\
k
\end{array}\right)+\left(\begin{array}{c}
n-1 \\
k-1
\end{array}\right)\right)\left(\begin{array}{c}
m+n-k-1 \\
m-k
\end{array}\right) \\
& =(-1)^{m+n-H}\left(\begin{array}{c}
H \\
m
\end{array}\right)\left(\begin{array}{c}
m \\
m+n-H
\end{array}\right)+L(m, n-1) \\
& +\sum_{k=0}^{m+n-H-2}(-1)^{k+1}\left(\begin{array}{c}
n-1 \\
k
\end{array}\right)\left(\begin{array}{c}
m+n-k-2 \\
m-1-k
\end{array}\right) \\
& =(-1)^{m+n-H}\left(\begin{array}{c}
H \\
m
\end{array}\right)\left(\begin{array}{c}
m \\
m+n-H
\end{array}\right)+L(m, n-1)-L(m-1, n-1) .
\end{aligned}
$$

Therefore,

$$
\begin{gathered}
L(m, n)-L(m-1, n)-L(m, n-1)+L(m-1, n-1) \\
=(-1)^{n+m-H}\left(\begin{array}{c}
H \\
m
\end{array}\right)\left(\begin{array}{c}
m \\
n+m-H
\end{array}\right) .
\end{gathered}
$$

Thus we see that (2.19) and (2.20) show that $L(m, n)$ and $R(m, n)$ satisfy the same bilinear recurrence. Since they have the same initial values, we have that $L(m, n)=R(m, n)$ for all nonnegative $n$ and $m$.

2.4. Existence of an Antipode. We now show that the linear map $S$ defined in Section 1 is an antipode on the bialgebra $\mathcal{A}_{\lambda}$, thus making the bialgebra into a Hopf algebra.

Since $A$ is commutative, we only need to prove

$$
\mu \circ(S \otimes \mathrm{id}) \circ \Delta=\eta \circ \varepsilon .
$$


From the definitions of $\varepsilon$ and $\eta$, we have

$$
\eta \circ \varepsilon\left(a_{n}\right)= \begin{cases}a_{0}, & n=0, \\ \lambda a_{0}, & n=1, \\ 0, & n>1 .\end{cases}
$$

Thus to prove that $S$ is an antipode on $A$, we only need to show that

$$
\mu\left(S \otimes \operatorname{id}\left(\Delta\left(a_{n}\right)\right)\right)= \begin{cases}a_{0}, & n=0 \\ \lambda a_{0}, & n=1, \\ 0, & n>1 .\end{cases}
$$

Recall that we have defined the $C$-linear map $S: A \rightarrow A$ by

$$
S\left(a_{n}\right)=(-1)^{n} \sum_{v=0}^{n}\left(\begin{array}{c}
n-3 \\
v-3
\end{array}\right) \lambda^{n-v} a_{v} .
$$

Using this and the definitions of $\mu$ and $\Delta$, we have

$$
\begin{aligned}
\mu(S & \left.\otimes \operatorname{id}\left(\Delta\left(a_{n}\right)\right)\right) \\
& =\sum_{k=0}^{n} \sum_{i=0}^{n-k} \sum_{v=0}^{i} \sum_{\ell=0}^{v}(-1)^{k+i} \lambda^{k+i-v+\ell}\left(\begin{array}{c}
i-3 \\
v-3
\end{array}\right)\left(\begin{array}{c}
n-k-i+v-\ell \\
v
\end{array}\right)\left(\begin{array}{l}
v \\
\ell
\end{array}\right) a_{n-k-i+v-\ell} .
\end{aligned}
$$

With a change of variable $\ell=n-k-i+v-w(w=n-k-i+v-\ell)$, we get

$$
\begin{aligned}
\mu(S & \left.\otimes \operatorname{id}\left(\Delta\left(a_{n}\right)\right)\right) \\
& =\sum_{k=0}^{n} \sum_{i=0}^{n-k} \sum_{v=0}^{i} \sum_{w=n-k-i}^{n-k-i+v}(-1)^{k+i} \lambda^{n-w}\left(\begin{array}{c}
i-3 \\
v-3
\end{array}\right)\left(\begin{array}{c}
w \\
v
\end{array}\right)\left(\begin{array}{c}
v \\
n-k-i+v-w
\end{array}\right) a_{w} .
\end{aligned}
$$

As in the proof of diagram (2.4), we want constant limits for the summations. Since $\left(\begin{array}{l}x \\ y\end{array}\right)=0$ for integers $x, y$ with $x \geq 0$ and either $y<0$ or $y>x$, we have

$$
\begin{gathered}
i>n-k \Rightarrow n-k-i<0 \\
\Rightarrow\left\{\begin{array}{c}
n-k-i+v-w<0 \Rightarrow\left(\begin{array}{c}
v \\
n-k-i+v-w
\end{array}\right)=0, \\
\left(\begin{array}{l}
w \\
v
\end{array}\right)=0, \\
w<n-k-i \quad \Rightarrow n-k-i-w>0 \\
\Rightarrow n-k-i+v-w>v \Rightarrow\left(\begin{array}{c}
v \\
n-k-i+v-w
\end{array}\right)=0 ;
\end{array}\right. \\
w>n-k-i+v \Rightarrow n-k-i+v-w<0 \\
\Rightarrow\left(\begin{array}{c}
v \\
n-k-i+v-w
\end{array}\right)=0 .
\end{gathered}
$$

Therefore, the last nested sum can be replaced by

$$
\begin{aligned}
& \sum_{k=0}^{n} \sum_{i=0}^{n} \sum_{v=0}^{i} \sum_{w=0}^{n}(-1)^{k+i} \lambda^{n-w}\left(\begin{array}{c}
i-3 \\
v-3
\end{array}\right)\left(\begin{array}{c}
w \\
v
\end{array}\right)\left(\begin{array}{c}
v \\
n-k-i+v-w
\end{array}\right) a_{w} \\
= & \sum_{w=0}^{n} \lambda^{n-w}\left[\sum_{k=0}^{n} \sum_{i=0}^{n} \sum_{v=0}^{i}(-1)^{k+i}\left(\begin{array}{c}
i-3 \\
v-3
\end{array}\right)\left(\begin{array}{c}
w \\
v
\end{array}\right)\left(\begin{array}{c}
v \\
n-k-i+v-w
\end{array}\right)\right] a_{w} .
\end{aligned}
$$


Thus to prove equation (2.21) we only need to prove

Theorem 2.4. For any integers $n \geq w \geq 0$, we have

$$
\begin{aligned}
& \sum_{k=0}^{n} \sum_{i=0}^{n} \sum_{v=0}^{i}(-1)^{k+i}\left(\begin{array}{c}
i-3 \\
v-3
\end{array}\right)\left(\begin{array}{l}
w \\
v
\end{array}\right)\left(\begin{array}{c}
v \\
n-k-i+v-w
\end{array}\right) \\
& = \begin{cases}1, & \text { if }(n, w)=(0,0) \text { or }(1,0), \\
0, & \text { otherwise. }\end{cases}
\end{aligned}
$$

Proof. The proof of this identity requires only three facts [14]:

$$
\begin{array}{r}
\left(\begin{array}{c}
-A \\
i
\end{array}\right)=(-1)^{i}\left(\begin{array}{c}
A+i-1 \\
i
\end{array}\right), \\
\sum_{j \geq 0}\left(\begin{array}{c}
A \\
j
\end{array}\right)\left(\begin{array}{c}
B \\
C-j
\end{array}\right)=\left(\begin{array}{c}
A+B \\
C
\end{array}\right), \\
\sum_{j=0}^{N}(-1)^{j}\left(\begin{array}{c}
N \\
j
\end{array}\right)= \begin{cases}1, & \text { if } N=0, \\
0, & \text { otherwise. }\end{cases}
\end{array}
$$

Hence we have that

$$
\begin{aligned}
& \sum_{k=0}^{n} \sum_{i=0}^{n} \sum_{v=0}^{i}(-1)^{k+i}\left(\begin{array}{c}
i-3 \\
v-3
\end{array}\right)\left(\begin{array}{c}
w \\
v
\end{array}\right)\left(\begin{array}{c}
v \\
n-k-i+v-w
\end{array}\right) \\
& =\sum_{k=0}^{n} \sum_{v=0}^{n} \sum_{i=v}^{n}(-1)^{k+i}\left(\begin{array}{c}
i-3 \\
v-3
\end{array}\right)\left(\begin{array}{c}
w \\
v
\end{array}\right)\left(\begin{array}{c}
v \\
n-k-i+v-w
\end{array}\right) \\
& \quad=\sum_{k=0}^{n} \sum_{v=0}^{n} \sum_{i=0}^{n-v}(-1)^{k+i+v}\left(\begin{array}{c}
i+v-3 \\
v-3
\end{array}\right)\left(\begin{array}{c}
w \\
v
\end{array}\right)\left(\begin{array}{c}
v \\
n-k-i-w
\end{array}\right) .
\end{aligned}
$$

Now by (2.23) and (2.24) we find that this sum equals

$$
\begin{aligned}
& \sum_{k=0}^{n} \sum_{v=0}^{n}(-1)^{k+v}\left(\begin{array}{c}
w \\
v
\end{array}\right) \sum_{i=0}^{n-v}\left(\begin{array}{c}
2-v \\
i
\end{array}\right)\left(\begin{array}{c}
v \\
n-k-i-w
\end{array}\right) \\
& \quad=\sum_{k=0}^{n} \sum_{v=0}^{n}(-1)^{k+v}\left(\begin{array}{c}
2 \\
n-k-w
\end{array}\right)\left(\begin{array}{c}
w \\
v
\end{array}\right) .
\end{aligned}
$$

By (2.25) we find that this sum equals

$$
\begin{aligned}
& \begin{cases}0, & \text { if } w>0, \\
\sum_{k=0}^{n}(-1)^{k}\left(\begin{array}{c}
2 \\
n-k
\end{array}\right), & \text { if } w=0\end{cases} \\
& = \begin{cases}0, & \text { if } w>0, \\
1, & \text { if } w=0 \text { and } n=0, \\
1, & \text { if } w=0 \text { and } n=1, \\
0, & \text { if } n>1 .\end{cases}
\end{aligned}
$$

This completes the proof of Theorem 1.1. 


\section{ISOMORPHISMS BETWEen $\lambda$-DIVIDED POWER Hopf ALGEBRAS}

We will prove the three statements in Theorem 1.2 by proving Propositions 3.1 3.2 and 3.4. To distinguish elements in $\lambda$-divided power Hopf algebras with various values of $\lambda$, we write

$$
A_{\lambda}=\bigoplus_{n \geq 0} C a_{\lambda, n}
$$

where $\left\{a_{\lambda, n}\right\}$ is the standard basis.

Proposition 3.1. Let $\lambda, \nu$ be in C. If $(\lambda)=(\nu)$, then $\mathcal{A}_{\lambda}$ and $\mathcal{A}_{\nu}$ are isomorphic Hopf algebras.

Proof. Suppose $(\lambda)=(\nu)$. Then $\lambda=\omega \nu$ for a unit $\omega$ in $C$. Define

$$
\varphi: A_{\lambda} \rightarrow A_{\nu}, a_{\lambda, n} \mapsto \omega^{n} a_{\nu, n} .
$$

Then it is straightforward to verify that $\varphi$ is a homomorphism of Hopf algebras. More precisely, we have

$$
\begin{aligned}
\varphi \circ \mu_{\lambda} & =\mu_{\nu} \circ(\varphi \otimes \varphi), \\
(\varphi \otimes \varphi) \circ \Delta_{\lambda} & =\Delta_{\nu} \circ \varphi, \\
\varphi \circ S_{\lambda} & =S_{\nu} \circ \varphi .
\end{aligned}
$$

It is also easily seen that $\varphi$ has an inverse given by sending $a_{\nu, n}$ to $\omega^{-n} a_{\lambda, n}$.

Proposition 3.2. Let $C$ be a $\mathbb{Q}$-algebra, and let $\lambda, \nu$ be in $C$. Then $\mathcal{A}_{\lambda}$ and $\mathcal{A}_{\nu}$ are isomorphic Hopf algebras if and only if $(\lambda)=(\nu)$.

Proof. By Proposition 3.1, we only need to prove that if $\mathcal{A}_{\lambda}$ and $\mathcal{A}_{\nu}$ are isomorphic Hopf algebras, then $(\lambda)=(\nu)$.

Suppose $C$ is a $\mathbb{Q}$-algebra and $\mathcal{A}_{\lambda} \cong \mathcal{A}_{\nu}$. Let $\varphi: \mathcal{A}_{\lambda} \rightarrow \mathcal{A}_{\nu}$ be a Hopf algebra isomorphism. By [6, Proposition 3.2], both $A_{\lambda}$ and $A_{\nu}$ are isomorphic to $C[x]$ as $C$ algebras with $a_{\lambda, 1}$ and $a_{\nu, 1}$ as the generators. It follows that $\varphi\left(a_{\lambda, 1}\right)=\sum_{i \geq 1} c_{i} a_{\nu, i}$ with $c_{1} \in C^{*}$. Likewise, $\varphi^{-1}\left(a_{\nu, 1}\right)=\sum_{i \geq 1} d_{i} a_{\lambda, i}$ with $d_{1} \in C^{*}$. Then we have

$$
\begin{aligned}
\left((\varphi \otimes \varphi) \circ \Delta_{\lambda}\right)\left(a_{\lambda, 1}\right) & =(\varphi \otimes \varphi)\left(1 \otimes a_{\lambda, 1}+a_{\lambda, 1} \otimes 1-\lambda \otimes 1\right) \\
& =1 \otimes\left(\sum_{i \geq 1} c_{i} a_{\nu, i}\right)+\left(\sum_{i \geq 1} c_{i} a_{\nu, i}\right) \otimes 1-\lambda \otimes 1 \\
& =-\lambda \otimes 1+\text { higher-degree terms }
\end{aligned}
$$

and

$$
\begin{aligned}
& \left(\Delta_{\nu} \circ \varphi\right)\left(a_{\lambda, 1}\right) \\
& =\Delta_{\nu}\left(\sum_{i \geq 1} c_{i} a_{\nu, i}\right) \\
& =c_{1}\left(1 \otimes a_{\nu, 1}+a_{\nu, 1} \otimes 1-\nu \otimes 1\right) \\
& +\quad c_{2}\left(1 \otimes a_{\nu, 2}+a_{\nu, 1} \otimes a_{\nu, 1}+a_{\nu, 2} \otimes 1-\nu\left(1 \otimes a_{\nu, 1}+a_{\nu, 1} \otimes 1\right)+\nu^{2} \otimes 1\right) \\
& +\cdots \\
& =-\nu\left(\sum_{i \geq 1}(-\nu)^{i-1} c_{i}\right)(1 \otimes 1)+\text { higher-degree terms. }
\end{aligned}
$$


Here the degree is the natural one given by the standard basis $a_{\nu, i}$. That is, the degree of $a_{\nu, i} \otimes a_{\nu, j}$ is $(i, j)$ with the lexicographic order. Since $\varphi$ is a Hopf algebra isomorphism, we have

$$
\lambda=\nu\left(\sum_{i \geq 1}(-\nu)^{i-1} c_{i}\right) .
$$

So $C \lambda \subseteq C \nu$. Considering $\varphi^{-1}$, we similarly get

$$
\nu=\lambda\left(\sum_{i \geq 1}(-\lambda)^{i-1} d_{i}\right) .
$$

So $C \nu \subseteq C \lambda$. Then $C \nu=C \lambda$.

For future reference, we record the following easy corollary of Proposition 3.2 .

Corollary 3.3. Let $C$ be a $\mathbb{Q}$-algebra and let $\nu$ be a unit in $C$. Then $\mathcal{A}_{\nu} \neq \mathcal{A}_{0}$.

Proposition 3.4. Let $\lambda$ and $\nu$ be in $C$. If either

(1) $\nu$ is not contained in $\sqrt{(\lambda)}$, or

(2) there is a prime number $p$ such that $\nu^{p-1}$ is not contained in $(\lambda, p)$,

then $\mathcal{A}_{\lambda}$ is not isomorphic to $\mathcal{A}_{\nu}$ as $C$-algebras.

The third statement of Theorem 1.2 follows from the first case of the proposition. To display the utility of the second case, we provide the following examples.

\section{Example 3.5.}

(1) Let $C=\mathbb{F}_{p}[x] /\left(x^{r}\right)$ with $r \geq p$. Then $\mathcal{A}_{\bar{x}} \neq \mathcal{A}_{0}$. Here $\bar{x}$ is the image of $x$ in $\mathbb{F}_{p}[x] /\left(x^{r}\right)$.

(2) Let $C=\mathbb{Z}[x] /\left(x^{r}\right)$ with $r \geq 2$. Then $\mathcal{A}_{\bar{x}} \nRightarrow \mathcal{A}_{0}$ (taking $p=2$ ).

Proof of Proposition 3.4. To consider the case when $\nu$ is not contained in $\sqrt{(\lambda)}$, we begin with a special situation.

Lemma 3.6. Let $C$ be reduced with characteristic $p$ for a prime number $p$. Let $\nu$ be a unit in $C$. Then $\mathcal{A}_{0} \neq \mathcal{A}_{\nu}$.

Proof. By [5. Theorem 4.8], the nilradical $N\left(A_{0}\right)$ of $A_{0}$ is $\bigoplus_{n \geq 1} C a_{0, n}$. By [5] Lemma 4.9], we have $N\left(A_{0}\right)^{p}=0$. So we only need to show that $A_{\nu}$ has no nonzero nilpotent element $x$ with $x^{p}=0$.

Let there be such an element $x \in A_{\nu}$. Write $x=\sum_{i=k}^{\infty} c_{i} a_{\nu, i}$ with $c_{k} \neq 0$. Then by the product formula in $A_{\nu}$ (see (ii) in the introduction),

$$
x^{p}=c_{k}^{p} a_{\nu, k}^{p}+\text { a term in } \bigoplus_{i=k+1}^{\infty} C a_{\nu, i}
$$

and

$$
a_{\nu, k}^{p}=\nu^{(p-1) k} a_{\nu, k}+\text { a term in } \bigoplus_{i=k+1}^{\infty} C a_{\nu, i} .
$$

Therefore,

$$
x^{p}=c_{k}^{p} \nu^{p-1} a_{\nu, k}+\text { a term in } \bigoplus_{i=k+1}^{\infty} C a_{\nu, i} .
$$

So we must have $c_{k}^{p} \nu^{p-1}=0$. Since $\nu$ is a unit, we have $c_{k}^{p}=0$. Since $C$ is reduced, we have $c_{k}=0$. This is a contradiction. 
Continuing with the proof of Proposition [3.4, we now suppose $\nu \notin \sqrt{(\lambda)}$, and $\mathcal{A}_{\lambda} \cong \mathcal{A}_{\nu}$. Then $\nu$ is not nilpotent and $(\lambda) \cap\left\{\nu^{n}\right\}$ is empty. By [11, Example 4], the image $\lambda / 1$ of $\lambda$ in the localization $\left\{\nu^{n}\right\}^{-1} C$ is not a unit. Let $P$ be a maximal ideal of $\left\{\nu^{n}\right\}^{-1} C$ containing $\lambda / 1$. Then the image $\tilde{\lambda}$ of $\lambda / 1$ (resp. $\widetilde{\nu}$ of $\left.\nu / 1\right)$ in the field $\left\{\nu^{n}\right\}^{-1} C / P$ is zero (resp. a unit). From $\mathcal{A}_{\lambda} \cong \mathcal{A}_{\nu}$, we have

$$
\left(\left\{\nu^{n}\right\}^{-1} C / P\right) \otimes_{C} A_{\lambda} \cong\left(\left\{\nu^{n}\right\}^{-1} C / P\right) \otimes_{C} A_{\nu} .
$$

That is, $\mathcal{A}_{\tilde{\lambda}} \cong \mathcal{A}_{\widetilde{\nu}}$. This is a contradiction by Corollary 3.3 (when the characteristic of the field $\left\{\nu^{n}\right\}^{-1} C / P$ is 0 ) and Lemma 3.6 (when the characteristic is a prime number $p$ ).

We next consider the case when there is a prime number $p$ such that $\nu^{p-1}$ is not contained in the ideal $(\lambda, p)$ of $C$. We again start with a special situation.

Lemma 3.7. Let $C$ be of characteristic $p$. If $\nu^{p-1}$ is not zero, then $\mathcal{A}_{\nu}$ is not isomorphic to $\mathcal{A}_{0}$.

Proof. Suppose $\mathcal{A}_{\nu}$ is isomorphic to $\mathcal{A}_{0}$. Let $\varphi: A_{0} \rightarrow A_{\nu}$ be an isomorphism of $C$ algebras. By [5] Theorem 4.8], the nilradical $N\left(A_{0}\right)$ of $A_{0}$ is $N(C) \oplus\left(\bigoplus_{n \geq 1} C a_{0, n}\right)$. On the other hand, by the product formula for $a_{\nu, n} \in A_{\nu}$ and the fact that $\bigoplus_{n \geq 1} C a_{\nu, n}$ is an ideal of $A_{\nu}$, we have $N\left(A_{\nu}\right)=N(C) \oplus L$ for an ideal $L$ of $A_{\nu}$ contained in $\bigoplus_{n \geq 1} C a_{\nu, n}$. Then $\varphi$ induces an isomorphism between the $C / N(C)$ algebras $A_{0} / N\left(A_{0}\right) \cong C / N(C)$ and $A_{\nu} / N\left(A_{\nu}\right) \cong C / N(C) \oplus\left(\bigoplus_{n \geq 1} C a_{\nu, n}\right) / L$. It follows that $L=\bigoplus_{n>1} C a_{\nu, n}$. Thus $a_{\nu, 1}$ is nilpotent. By [5, Lemma 4.9], $N\left(A_{0}\right)^{p}$ is contained in $N(C)^{p}$. So we must have $a_{\nu, 1}^{p} \in N(C)^{p}$. In particular, $a_{\nu, 1}^{p} \in C$. But this cannot be true since

$$
a_{\nu, 1}^{p}=\nu^{p-1} a_{\nu, 1}+\text { a term in } \bigoplus_{i=k+1}^{\infty} C a_{\nu, i}
$$

and $\nu^{p-1} \neq 0$.

In the general case, consider $\bar{C}=C /(\lambda, p)$ and let $\bar{\lambda}$ (resp. $\bar{\nu}$ ) be the image of $\lambda$ (resp. $\nu$ ) in $\bar{C}$. Then by Lemma $3.7, \mathcal{A}_{\bar{\nu}} \approx \mathcal{A}_{\bar{\lambda}}$. So $\mathcal{A}_{\nu} \approx \mathcal{A}_{\lambda}$.

\section{ACKNOWLEDGEMENTS}

We thank Jacob Sturm for helpful discussions.

\section{REFERENCES}

[1] G. Baxter, An analytic problem whose solution follows from a simple algebraic identity, Pacific J. Math. 10 (1960), 731-742. MR 22:9990

[2] P. Berthelot and A. Ogus, Notes on Crystalline Cohomology, Princeton University Press, Princeton, NJ, 1978. MR 58:10908

[3] P. Cartier, On the structure of free Baxter algebras, Adv. in Math. 9 (1972), 253-265. MR 49:2807

[4] K.-T. Chen, Integration of paths, geometric invariants and a generalized Baker-Hausdorff formula, Ann. of Math. 65 (1957), 163-178. MR 19:12a

[5] L. Guo, Properties of free Baxter algebras, Adv. in Math. 151 (2000), 346-374. MR 2001f: 16048

[6] L. Guo, Baxter algebras and the umbral calculus, Adv. in Appl. Math. 27 (2001), 405-426. MR 2003b:05020

[7] L. Guo, Baxter algebras and differential algebras, In: Differential Algebra and Related Topics, World Scientific Publishing, River Edge, NJ, 2002, pp. 281-305. 
[8] L. Guo and W. Keigher, Baxter algebras and shuffle products, Adv. in Math. 150 (2000), 117-149. MR 2001g:05015

[9] L. Guo and W. Keigher, On free Baxter algebras: completions and the internal construction, Adv. in Math. 151 (2000), 101-127. MR 2001c: 16046

[10] W. Keigher, On the ring of Hurwitz series, Comm. Algebra 25 (1997), 1845-1859. MR 98e: 13022

[11] H. Matsumura, Commutative Ring Theory, Cambridge University Press, 1994. MR 90i:13001

[12] W. Nichols and M. Sweedler, Hopf algebras and combinatorics, In: Umbral Calculus and Hopf Algebras, Contemporary Mathematics 6, Amer. Math. Soc., Providence, RI, 1982, pp. 49-84. MR 83g:16019

[13] R. Ree, Lie elements and an algebra associated with shuffles, Ann. Math. 68 (1958), 210-220. MR 26:6447

[14] J. Riordan, Combinatorial Identities, John Wiley and Sons, New York, 1968. MR 38:53

[15] S. Roman, The Umbral Calculus, Pure and Applied Mathematics 111, Academic Press, Orlando, FL, 1984. MR 87c:05015

[16] S. Roman and G.-C. Rota, The umbral calculus, Adv. Math. 27 (1978), 95-188. MR 58:5256

[17] G.-C. Rota, Baxter algebras and combinatorial identities. I, II, Bull. Amer. Math. Soc. 75 (1969), 325-329, 330-334. MR 39:5387

[18] G.-C. Rota, Baxter operators, an introduction, In: Gian-Carlo Rota on Combinatorics, Introductory Papers and Commentaries, Joseph P. S. Kung, editor, Birkhäuser, Boston, 1995, pp. 504-512. MR 99b:01027

[19] G.-C. Rota, Ten mathematics problems I will never solve, Invited address at the joint meeting of the American Mathematical Society and the Mexican Mathematical Society, Oaxaca, Mexico, December 6, 1997, Mitt. Dtsch. Math.-Ver., Heft 2, 1998, pp. 45-52.

[20] D. Stanton and D. White, Constructive Combinatorics, Springer-Verlag, New York, 1986. MR 88a:05001

Department of Mathematics, Pennsylvania State University, University Park, PennSYLVANIA 16802

E-mail address: andrews@math.psu.edu

Department of Mathematics and Computer Science, Rutgers University at Newark, NEWARK, NEW JERSEy 07102

E-mail address: liguo@newark.rutgers.edu

Department of Mathematics and Computer Science, Rutgers University at Newark, NewARK, New JeRSEy 07102

E-mail address: keigher@newark.rutgers.edu

Department of Mathematics, University of Wisconsin, Madison, Wisconsin 53706

E-mail address: ono@math.wisc.edu 